\title{
Trend analysis of nasal bone fracture
}

\author{
Kwang Seog Kim ${ }^{1}$, \\ Han Gyeol Lee', \\ Jun Ho Shin', \\ Jae Ha Hwang ${ }^{1}$, \\ Sam Yong Lee ${ }^{1}$ \\ Departments of ${ }^{1}$ Plastic and \\ Reconstructive Surgery and ${ }^{2}$ Preventive \\ Medicine, Chonnam National \\ University Medical School, Gwangju, \\ Korea
}

\begin{abstract}
Background: Nasal bone fractures occur frequently because the nasal bone is located at the forefront of the face. The goal of this study was to examine the cause, change in severity, change in incidence, and demographics of nasal bone fracture according to today's lifestyle.

Methods: A total of 2,092 patients diagnosed as having nasal bone fractures at our department between 2002 and 2017 were included in this study. We retrospectively examined patients' medical records to extract information regarding age, sex, cause of injury, combined facial bone fractures, and related injuries such as skull base fracture, spinal cord injury, brain hemorrhage, and other bone fractures. Fracture severity was classified by nasal bone fracture type.

Results: No statistically significant difference was found in annual number of patients treated for nasal bone fracture. The proportion of patients who underwent closed reduction was significantly decreased over time for those with nasal bone fractures caused by traffic accidents. However, it was not significantly changed for those with nasal bone fractures due to other causes. The number of patients with combined facial bone fractures increased over time. Incidences of severe nasal bone fracture also increased over time.

Conclusion: The study suggested that there is a decrease in the frequency and increase in the severity of nasal bone fracture due to traffic accident. Many protective devices prevent nasal bone fractures caused by a small amount of external force; however, these devices are not effective against higher amounts of external force. This study highlights the importance of preoperative thorough evaluation to manage patients with nasal bone fractures due to traffic accident.
\end{abstract}

Keywords: Nasal surgery / Nose / Nasal bone

\section{INTRODUCTION}

The nasal bone-cartilage framework includes an immobile bony pyramid, cartilage, and nasal septum. These components support the nose and help maintain airway patency. Both cartilaginous and bony components are susceptible to trauma [1]. The nose is located in the middle of the human face. It is an important structure in aesthetics [2]. The nasal bone is located at the top of the nose and supports the three-dimensional structure of the nose. As such, the nasal bone is located at the fore-

\section{Correspondence: Kwang Seog Kim}

Department of Plastic and Reconstructive Surgery, Chonnam National University

Medical School, 42 Jebong-ro, Dong-gu, Gwangju 61469, Korea

E-mail: pskim@chonnam.ac.kr

Received October 16, 2018 / Accepted October 24, 2018 front of the face and vulnerable to external trauma. Fractures can occur due to many different causes, including traffic accidents, assault, external trauma from other objects, and falls. Such fractures require accurate initial treatment and early on diagnosis. If necessary, closed reduction should be performed. Failure to take proper management could result in deformity and functional discomfort. Several protocols have been developed to treat patients with nasal bone fractures accordingly. Many lifestyle-related changes have occurred recently. For example, wearing seat belts is more strictly enforced by law. In addition, various wearable pieces of protective gear during exercise have been developed. Society's interest in safety is increasing. Therefore, the frequency and extent of injuries are decreasing. Here, changes in incidence of nasal bone fracture, the most 
common facial injury, as a result of such lifestyle changes were determined.

\section{METHODS}

A total of 2,092 patients diagnosed as having nasal bone fractures at our department between 2002 and 2017 were included in this study. We retrospectively examined patients' medical records. Major variables were sex, age, year and type of fracture type, combined facial bone fractures, additional body fractures, and cause of injury. Statistical analysis was conducted using linear regression and linear-by-linear association test with IBM SPSS ver. 22.0 (IBM Corp., Armonk, NY, USA). Data were not pre-processed for statistical analysis. To analyze changes in fracture patterns, an analysis was conducted for three groups divided according to the year of fracture occurrence (group A, 2002-2006; group B, 2007-2012; and group C, 2013-2017).

We classified patients with nasal bone fractures into four groups using the existing known classification method as follows (Fig. 1) [3]: type I, linear fracture without depression; type

Type of fracture

Type II
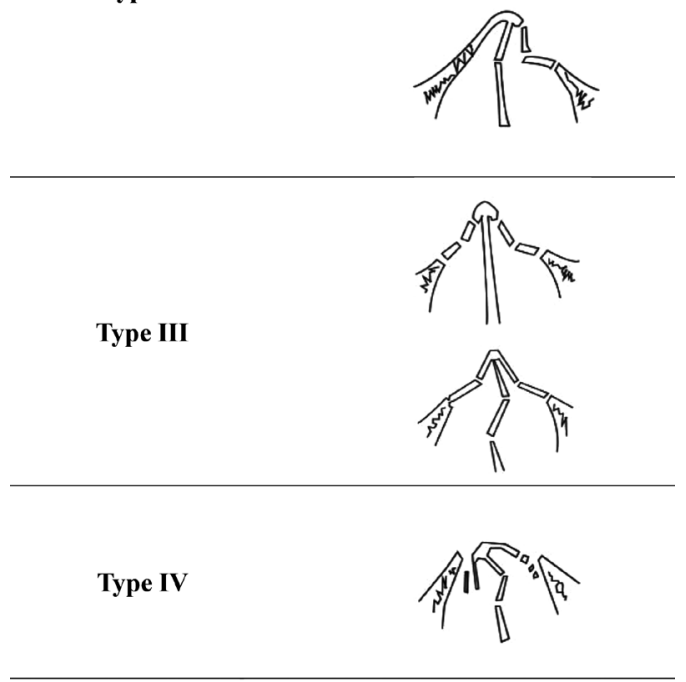

Fig. 1. Nasal bone fracture types [3]. Type I, linear fracture without depression; Type II, unilateral depression with or without septal fracture; Type III, bilateral depression with or without septal fracture; Type IV, comminuted fracture.
II, unilateral depression with or without septal fracture; type III, bilateral depression with or without septal fracture; type IV, comminuted fracture. We studied nasal bone fractures according to the following cause: traffic accident, assault, fall, or other traumas. First, we conducted a linear-by-linear association to test tendencies of increase and decrease for incidence of nasal bone fracture, depending on the cause over time. Next, we analyzed differences in nasal bone fracture severity with time by cause of fracture.

\section{RESULTS}

Trend analysis of the incidence of nasal bone fracture over time according to cause of injury

\section{Proportion of the incidence of nasal bone fracture by cause}

We conducted an analysis of linear-by-linear association with the value for time on the $\mathrm{X}$-axis and the number of occurrences of nasal bone fracture by different causes. We found that the incidence rate of nasal bone fracture caused by traffic accident decreased significantly ( $p=0.228$ ) (Fig. 2).

\section{Year and occurrence of nasal bone fracture cause}

Odds ratios were also analyzed to elucidate the relationship with other causes. The incidence of nasal bone fracture caused by traffic accident was higher than that of nasal bone fracture by other causes. It decreased significantly over time. The incidence of nasal bone fracture caused by trauma did not increase or decrease significantly over time compared to that of nasal

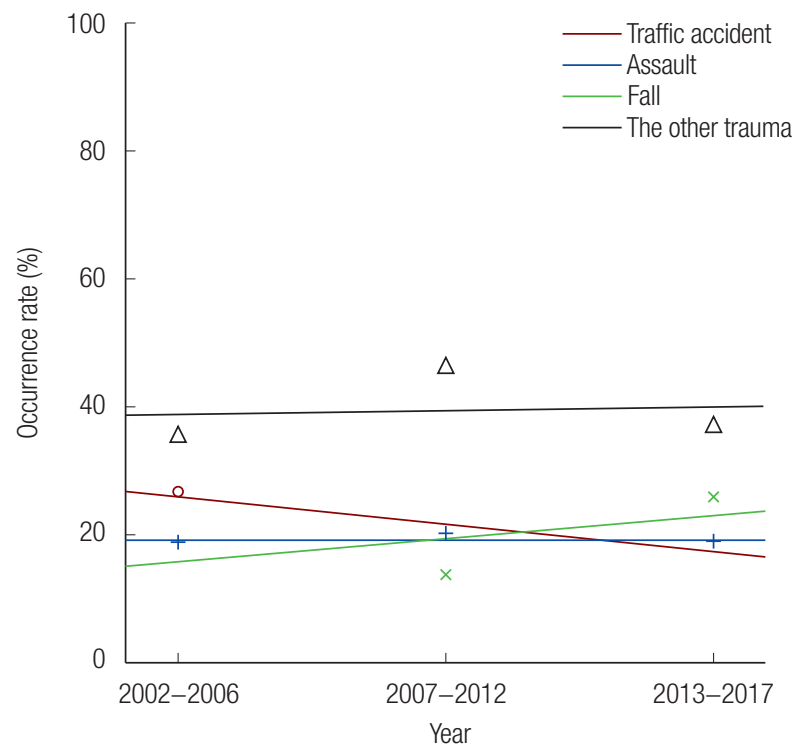

Fig. 2. Proportion of frequency of nasal bone fracture by mechanism. The $p$-values of regression slope for traffic accident are 0.228 , for assault, 0.981; for fall, 0.619; and for trauma, 0.928 . 
bone fracture by other causes. The incidence of nasal bone fracture caused by assault did not increase or decrease significantly over time. However, the incidence of nasal bone fracture caused by fall was significantly increased overtime. For survey groups in which the cause of injury was fall, the odds ratio showed a tendency of increase over time (Table 1).

\section{Trend analysis of nasal bone fracture severity over time} by cause of injury

Analysis between year and fracture severity, between occurrence and number of combined facial bone fractures and type of nasal bone fracture

In linear-by-linear association analysis, the increase in the occurrence of combined facial bone fractures was statistically significant. The linear association analysis revealed that the increase in the number of combined facial bone fractures over time was statistically significant. Regarding nasal bone fracture types, an overall increasing tendency was observed for types III and IV (Table 2).

\section{Traffic accident group}

In the survey for patients with fractures caused by traffic accident $(n=456)$, the increase in the occurrence of combined facial bone fractures over time was statistically significant. In addition, the number of combined facial bone fractures increased over time. However, there was no significant change in nasal bone fracture type.

\section{Assault group}

The incidence of related injuries in patients with fractures caused by assault $(n=404)$ did not change significantly. No significant change was observed in the occurrence or the number of combined facial bone fractures or nasal bone fracture type.

\section{Fall group}

For patients who had fractures caused by fall $(n=405)$, the decrease in the incidence of related injuries over time was significant, while the incidence of associated fractures increased over time. The number of combined other facial bone fractures in-

Table 1. Linear-by-linear association between year and occurrence of nasal bone fracture by cause

\begin{tabular}{|c|c|c|c|c|c|c|}
\hline Variable & Subgroup & №. (\%) & Group A & Group B & Group C & $p$-value \\
\hline Total & & $2,092(100)$ & 708 (33.8) & $731(34.9)$ & $653(31.2)$ & \\
\hline \multirow[t]{2}{*}{ Traffic accident } & Others & $1,636(78.2)$ & $519(31.7)$ & $585(35.8)$ & $532(32.5)$ & $<0.001^{*}$ \\
\hline & Traffic accident & $456(21.8)$ & $189(41.4)$ & $146(32.0)$ & $121(26.5)$ & \\
\hline \multirow[t]{2}{*}{ Assault } & Others & $1,688(80.7)$ & $575(34.1)$ & $583(34.5)$ & $530(31.4)$ & 0.966 \\
\hline & Assault & 404 (19.3) & $133(32.9)$ & $148(36.6)$ & $123(30.4)$ & \\
\hline \multirow[t]{2}{*}{ Fall } & Others & $1,687(80.6)$ & $573(34.0)$ & $630(37.3)$ & $484(28.7)$ & $0.002^{*}$ \\
\hline & Fall & 405 (19.4) & 135 (33.3) & $101(24.9)$ & $169(41.7)$ & \\
\hline \multirow[t]{2}{*}{ Other trauma } & Others & $1,265(60.5)$ & $457(36.1)$ & $395(31.2)$ & 413 (32.6) & 0.551 \\
\hline & Trauma & 827 (39.5) & 251 (30.4) & $336(40.6)$ & $240(29.0)$ & \\
\hline
\end{tabular}

Group A, 2002-2006; Group B, 2007-2012; Group C, 2013-2017. Year ranges are expressed as number and percent (computed column-wise). The $p$-values were computed using a linear-by-linear association test, and number (\%) values were computed in the total sample or subgroups excluding missing data. Odds ratio and $95 \%$ confidence intervals were computed in the subsample of cause (others vs. each cause). The decreased tendency of the occurrence of nasal bone fractures caused by traffic accident over time was statistically significant. The incidence of nasal bone fracture in patients who had a fall increased over time. ${ }^{*} p<0.05$

Table 2. Linear-by-linear association between year and fracture severity

\begin{tabular}{|c|c|c|c|c|c|c|}
\hline Classification & Subgroup & №. $(\%)$ & Group A & Group B & Group C & $p$-value \\
\hline Total & & $2,092(100)$ & $708(33.8)$ & $731(34.9)$ & $653(31.2)$ & \\
\hline \multirow[t]{2}{*}{ Presence of combined fracture } & No & $1,612(77.1)$ & $611(37.9)$ & $517(32.1)$ & $484(30.0)$ & $<0.001^{*}$ \\
\hline & Yes & $480(22.9)$ & 97 (20.2) & $214(44.6)$ & $169(35.2)$ & \\
\hline \multirow{3}{*}{$\begin{array}{l}\text { No. of combined facial bone } \\
\text { fractures }\end{array}$} & No & $1,612(77.1)$ & $611(37.9)$ & $517(32.1)$ & $484(30.0)$ & $<0.001^{*}$ \\
\hline & One & $393(18.8)$ & 76 (19.3) & $174(44.3)$ & $143(36.4)$ & \\
\hline & Two or more & $87(4.2)$ & $21(24.1)$ & $40(46.0)$ & $26(29.9)$ & \\
\hline \multirow{4}{*}{ Fracture type } & I & $514(24.6)$ & $122(23.7)$ & $218(42.4)$ & $174(33.9)$ & $<0.001^{*}$ \\
\hline & $\|$ & $1,106(52.9)$ & $497(44.9)$ & $329(29.8)$ & $280(25.3)$ & \\
\hline & III & $300(14.3)$ & $66(22.0)$ & $110(36.8)$ & $124(41.3)$ & \\
\hline & IV & $172(8.2)$ & $23(13.4)$ & $74(43.0)$ & 75 (43.5) & \\
\hline
\end{tabular}

Group A, 2002-2006; group B, 2007-2012; and group C, 2013-2017. Year ranges are expressed as sample number and percent (computed column-wise). The p-values were computed using a linear-by-linear association test, and number (\%) values were computed in the total sample or subgroups excluding missing data. The decrease in the incidence of related injuries was statistically significant. The number and severity of facial bone fractures and occurrence of concomitant facial bone fracture were aggravated. ${ }^{*} p<0.05$. 
creased, while the number of nasal bone fracture type cases had a decreasing tendency.

\section{Other-trauma group}

Patients in the other-trauma group $(n=827)$ tended to have fewer injuries over time. The incidence of combined facial bone fractures increased, while the number of combined facial bone fractures tended to increase.

\section{DISCUSSION}

Nasal bone fractures are commonly observed in clinical settings. Although nasal bone fractures are considered minor injuries, the nasal bone is highly important in facial aesthetics. When a patient visits a hospital with a nasal bone fracture, radiography and facial computed tomography are performed. Although the patient's condition is taken into consideration, closed reduction is used as treatment $[4,5]$.

According to one study, the overall deformity rate was $10.4 \% \pm 4.8 \%$. No significant differences were found between patients who underwent closed reduction $(14.7 \% \pm 7.3 \%)$ and those who underwent open reduction $(9.4 \% \pm 4.4 \%)$. It was interesting that one-tenth of patients with nasal bone fractures experienced postoperative deformity (10.4\%), septal deviation (10.0\%), or nasal obstruction (10.5\%) after the operation. This pattern may be referred to as the "rule of 10" [5].

The cause of nasal bone fracture has been variously reported. The most common cause of nasal bone fracture was reported as traffic accidents in one study and motor cycle accidents in another study [6,7]. However, another study reported that assaults accounted for 38\%, falls accounted for 31\%, and accidents during exercise accounted for $17 \%$ of fractures [8]. A recently published study on juvenile patients in suburban areas has suggested that pedestrian traffic accidents is the most common cause of nasal bone fractures, followed by sport-related trauma. In urban areas, nasal bone fractures in patients in their teens were most commonly caused by accidental fall. However, in adult patients, regardless of their habitation, assault and vehicular traffic accident were the most common causes of nasal bone fractures [9]. According to ages of patients with nasal bone fractures, one study reported that nasal bone fracture was the most common among subjects in their 20s [10]. Another study stated that nasal bone fracture often occurs in people aged 11-20 years [3].

In a previous study, the most important independent cause of nasal bone fractures was traffic accidents while trauma caused by vehicular accident accounted for the largest part of nasal bone fractures. Recently, lifestyles have been changing rapidly.
The legislation of seat-belt wearing is one of these changes. In case of a traffic accident, the patient can be physically impacted by the anterior part of the windshield, steering wheel, instrument panel, and other structures of the vehicle. The face, especially the midface, is the most vulnerable one to such an environment. In one study about traffic accidents of 226 patients, half of these patients were injured in the midface, and $30 \%$ of all patients had nasal bone fractures [11]. Although the airbag could reduce the total force, it could only reduce force exerted on the zygoma, maxilla, and orbital wall. It had little effect on the force exerted on the nasal bone [12]. The speed gap of 13 $\mathrm{km} / \mathrm{hr}$ was required for the same injury in the face between the seat belt- and non-seat belt-wearing patients. Patients who did not wear their seat belts had facial damage at speeds of $>33$ $\mathrm{km} / \mathrm{hr}$. However, patients who wore their seat belts did not incur facial injuries at speeds of $<43.4 \mathrm{~km} / \mathrm{hr}$. At speeds of $>55.7$ $\mathrm{km} / \mathrm{hr}$, the incidence of facial injury increased [13].

In this study, the incidence of nasal bone fracture caused by traffic accidents decreased compared with that of nasal bone fracture caused by other mechanism. However, in patients with nasal bone fractures caused by traffic accidents, incidence rates of type III and IV nasal bone fractures increased. Although type II fractures showed a tendency to decrease compared to type I fractures, nasal bone fracture severity was increased. Patients had combined other facial bone fractures more frequently. These results were similar to those for the whole patient group.

The limitation of this study is a single trauma center-based study. There was a need to distinguish the patients according to whether they were pedestrian traffic accident or in car traffic accident. In addition, analysis of the traffic accident according to the type of vehicle will be needed. The study suggested that there is a decrease in the frequency and increase in the severity of nasal bone fracture due to traffic accident. Many protective devices prevent nasal bone fractures caused by a small amount of external force; however, these devices are not effective against higher amounts of external force. This study highlights the importance of preoperative thorough evaluation to manage patients with nasal bone fractures due to traffic accident.

\section{CONFLICT OF INTEREST}

KSK, a member of the Archives of Craniofacial Surgery, is the corresponding author of this article. However, he played no role whatsoever in the editorial evaluation of this article or the decision to publish it. Except for that, no potential conflict of interest relevant to this article was reported. 


\section{ORCID}

Kwang Seog Kim https://orcid.org/0000-0002-6766-4640

Han Gyeol Lee https://orcid.org/0000-0001-7835-0172

Jun Ho Shin https://orcid.org/0000-0002-0712-2794

Jae Ha Hwang https://orcid.org/0000-0001-7556-3594

Sam Yong Lee https://orcid.org/0000-0002-3185-2519

\section{REFERENCES}

1. Chan J, Most SP. Diagnosis and management of nasal fractures. Oper Tech Otolayngol Head Neck Surg 2008;19:263-6.

2. Rohrich RJ, Adams WP Jr. Nasal fracture management: minimizing secondary nasal deformities. Plast Reconstr Surg 2000; 106:266-73.

3. Kim JH, Lee JW, Park CH. Cosmetic rhinoseptoplasty in acute nasal bone fracture. Otolaryngol Head Neck Surg 2013;149: 212-8.

4. Mohammadi A, Ghasemi-Rad M. Nasal bone fracture: ultrasonography or computed tomography? Med Ultrason 2011;13: 292-5.

5. Cannon CR, Cannon R, Young K, Replogle W, Stringer S, Gasson E. Characteristics of nasal injuries incurred during sports activities: analysis of 91 patients. Ear Nose Throat J 2011;90:E812.
6. Hong HS, Cha JG, Paik SH, Park SJ, Park JS, Kim DH, et al. High-resolution sonography for nasal fracture in children. AJR Am J Roentgenol 2007;188:W86-92.

7. Fattahi T, Steinberg B, Fernandes R, Mohan M, Reitter E. Repair of nasal complex fractures and the need for secondary septo-rhinoplasty. J Oral Maxillofac Surg 2006;64:1785-9.

8. Hwang K, You SH, Kim SG, Lee SI. Analysis of nasal bone fractures; a six-year study of 503 patients. J Craniofac Surg 2006;17:261-4.

9. Liu C, Legocki AT, Mader NS, Scott AR. Nasal fractures in children and adolescents: mechanisms of injury and efficacy of closed reduction. Int J Pediatr Otorhinolaryngol 2015;79:223842.

10. Ashoor AJ, Alkhars FA. Nasal bone fracture. Saudi Med J 2000; 21:471-4.

11. Hitosugi M, Mizuno K, Nagai T, Tokudome S. Analysis of maxillofacial injuries of vehicle passengers involved in frontal collisions. J Oral Maxillofac Surg 2011;69:1146-51.

12. Simoni P, Ostendorf R, Cox AJ 3rd. Effect of air bags and restraining devices on the pattern of facial fractures in motor vehicle crashes. Arch Facial Plast Surg 2003;5:113-5.

13. Todorovic M, Vukcevic B, Cabarkapa M, Vukcevic N, Boljevic T, Radojevic N. The assessment of airbag deployment and seatbelt use in preventing facial injuries. Forensic Sci Med Pathol 2018;14:503-8. 